\title{
Caractérisation de l'état trophique du sanglier, en milieu forestier, à partir du gras mésentérique et des paramètres sanguins
}

\author{
R. MAUGeT, A. AUMAITRE $\left({ }^{*}\right)$, Y. PEIGNIER $\left({ }^{*}\right)$
}

Centre d'Etudes Biologiques des Animaux Sauvages, C.N.R.S. 79360 Beauvoir-sur-Niort, France.

(*) Station de Recherches Porcines, I.N.R.A.

St-Gilles, 35590 L'Hermitage, France.

Summary. Determination of the nutritional status of wild boars in woodland areas on the basis of mesenteric fat and blood parameters.

Blood samples as well as mesenteric fat for measurement of biochemical parameters were taken from 51 wild boars ( 36 males, 15 females) slaughtered over a period of one year. The relative weight of the mesenteric adipose tissue as well as its lipid content showed a maximum in the autumn and regularly decreased until the end of the next summer. The lipid content thus ranged from 39 to less than $10 \%$ at the end of the summer.

Variations in the blood levels of some metabolites measured in the plasma were compared with the nutritional status of the animals. The values showed a high glycaemia $(158 \mathrm{mg} / \mathrm{dl})$ that varied little with the season. In contrast, the cholesterol level decreased from the autumn till late winter with a minimum value in March ( 89 to $50 \mathrm{mg} / \mathrm{dl}$ ). The level of total proteins, which was low from February to September, increased during the autumn-winter period, independently of the albumin content which seemed to be constant. The urea content was highly variable thus masking possible seasonal variations. The creatinine level showed a minimum in the autumn $(2.0 \mathrm{mg} / \mathrm{dl})$ and a maximum in the summer $(2.9 \mathrm{mg} / \mathrm{dl})$, a period with little abundant food supplies.

Thus, the wild boar exhibits large seasonal variations of its metabolism and constitutes body reserves which are mobilised during periods of reduced food availability. The mesenteric fat weight as well as the blood levels of cholesterol and creatinine seem to characterize rather well the nutritional status of this animal.

\section{Introduction.}

L'établissement de profils biochimiques est une mesure de routine pour caractériser l'état métabolique et nutritionnel chez l'homme. Chez les animaux domestiques, on a proposé d'utiliser les variations des taux de constituants sanguins pour décrire une situation de couverture des besoins (glycémie, aminoacidémie) ou, à l'inverse, des situations de jeûne ou de catabolisme (urée, acides gras libres, glycérol). Les bovins et les ovins ont été les plus étudiés (Payne et al., 1970; Smith et al., 1978). De nombreuses valeurs de référence ont été 
apportées pour l'espèce porcine (Ursache et al., 1979 ; Benjaminsen et Dishington, 1981 ; Doornenball et al., 1983, 1986). Mais l'alimentation intensive chez ces animaux ne permet pas de déceler des variations saisonnières marquées.

La signification des variations biochimiques plasmatiques est plus spécialement évidente lors des carences notoires de la ration. Ainsi, chez le Porc, Tumbleson (1972) a montré que l'on pouvait caractériser l'état de sous-nutrition protéique engendré par une ration carencée. C'est ainsi qu'une forte carence en protéines de la ration entraîne des variations importantes du taux de protéines totales, de l'albumine et, dans une moindre mesure, de la créatinine. Par ailleurs, les animaux monogastriques, encore plus sensibles que les ruminants aux périodes de disette ou de lactation, mobilisent leurs réserves adipeuses internes. Au contraire, en situation d'abondance alimentaire, les suidés déposent, principalement dans la panne et le mésentère, des quantités importantes de lipides.

L'intérêt d'étendre aux animaux sauvages les méthodes d'investigation biochimiques est évident, la gestion des populations impliquant nécessairement la connaissance de l'état trophique des individus. Les populations de Cervidés ont ainsi été particulièrement étudiées (Seal et al., 1978; Warren et al., 1981) de même que les Pécaris (Lochmiller et al., 1985, 1986). Les données portant sur le Sanglier sont peu nombreuses (Singer et Ackerman, 1981 ; Barrat et al., 1984) et n'abordent pas l'aspect saisonnier des variations de l'état nutritionnel.

Le but de notre étude a été d'essayer de mettre en parallèle les variations de l'état trophique caractérisées au niveau des réserves graisseuses et des paramètres sanguins biochimiques.

\section{Matériel et méthodes.}

Animaux, échantillonnage, prélèvements. - Cette étude a été réalisée sur la population sauvage de Sangliers de la Réserve Nationale de Chizé (DeuxSèvres). Dans le cadre de la gestion des populations effectuée par l'Office National de la Chasse, nous avons pu disposer d'animaux capturés à différentes périodes de l'année. Nous n'avons considéré ici que les animaux ayant atteint ou dépassé l'âge de 8-10 mois (âge estimé suivant les critères d'évolution de la dentition définis par Matschke, 1967). Un échantillon de 51 animaux adultes ( 36 mâles et 15 femelles ; poids moyen : $57,6 \pm 5,6 \mathrm{~kg}$ ) a ainsi été retenu. La distribution mensuelle des poids vifs moyens des animaux est reportée dans le tableau 1. Le poids moyen varie peu, sauf pour les prélèvements effectués en avril/mai qui comportent une proportion de $50 \%$ d'animaux nés au printemps précédent.

Les animaux étudiés ont tous été piégés de nuit (au cours de leur phase d'activité nocturne) dans des enclos-pièges appâtés par des céréales. II est difficile de préciser le moment exact de la prise alimentaire. A l'abattage qui a lieu tôt le matin suivant la capture, il ne peut être que vérifié (d'après le contenu stomacal) qu'il y a eu prise alimentaire quelques heures auparavant. 
TABLEAU 1

Poids corpore/s moyens des animaux abattus.

\begin{tabular}{lcrc}
\hline & $\begin{array}{c}\text { Poids } \\
\mathrm{kg} \pm \mathrm{SE}\end{array}$ & $\mathbf{n}$ & $\begin{array}{c}\text { Effectifs } \\
\text { (mâles; femelles) }\end{array}$ \\
\hline Octobre/novembre & $63,8 \pm 4,8$ & 10 & $(7 ; 3)$ \\
Décembre/janvier & $50,8 \pm 5,4$ & 12 & $(8 ; 4)$ \\
Février/mars & $59,3 \pm 6,1$ & 3 & $(3 ; 0)$ \\
Avril/mai & $38,9 \pm 5,6$ & 14 & $(8 ; 6)$ \\
Juin/juillet & $52,0 \pm 7,9$ & 5 & $(4 ; 1)$ \\
Août/septembre & $62,8 \pm 6,5$ & 7 & $(66 ; 1)$ \\
\hline Moyenne pondérée & $57,6 \pm 5,6$ & 51 & \\
\hline
\end{tabular}

Les prélèvements sanguins sont effectués sur héparine. Le sang est centrifugé dans I'heure et le plasma recueilli est conservé à $-25^{\circ} \mathrm{C}$. Le tissu mésentérique intestinal, correspondant à une masse composite de vaisseaux, ganglions lymphatiques et tissu adipeux, est disséqué et pesé à l'état frais. Il est conservé à $-25^{\circ} \mathrm{C}$ pour détermination ultérieure de sa composition après broyage total, lyophilisation et homogénéisation.

Analyses biochimiques. - Les lipides du gras mésentérique sont déterminés par une extraction à froid dans le mélange méthanol-chloroforme-eau ; les résultats sont rapportés à la matière sèche du tissu disséqué. Les acides gras de l'extrait lipidique sont déterminés par chromatographie en phase gazeuse; les résultats sont exprimés en pourcentages respectifs des principaux acides gras et par l'indice d'insaturation utilisé par Girard et al. (1988) représenté par le rapport $\Sigma$ (\% de chaque acide gras insaturé $\times$ nombre de doubles liaisons) $/ \Sigma$ (\% de chaque acide gras insaturé). Le glucose est dosé par la méthode enzymatique de la glucose oxydase-peroxydase. Les protéines totales sont mesurées selon la méthode du biuret et la sérumalbumine selon la méthode au vert de bromocrésol. Le glycérol est mesuré par la méthode enzymatique de la glycérokinase en présence d'ATP, de phosphokinase et de lactate déshydrogénase, la créatinine par la méthode de Jaffe au picrate alcalin. Le cholestérol est déterminé grâce à la cholestérol-estérase-oxydase en association avec la peroxydase ; enfin, l'urée est dosée par le système uréase-glutamate déshydrogénase. L'ensemble des opérations et lectures est réalisé sur un Analyseur Biochimique Monoparamétrique automatique Isamat (ISAbiologie, Cachan).

Présentation des données, analyse statistique. - Les valeurs des différents paramètres mesurés ont été groupées sur des périodes de deux mois. Les données sont exprimées en moyennes et erreurs standards de la moyenne ( \pm SEM). Les moyennes correspondant à de faibles effectifs sont signalées dans les figures afin de les relativiser. La signification des différences entre les périodes a été évaluée en utilisant le test $t$ de Student. 


\section{Résultats.}

\section{Réserves adipeuses.}

Le poids frais du gras mésentérique est corrélé positivement avec le poids corporel de l'animal $(r 2=0,45)$. Du fait de la dispersion du poids corporel de l'échantillonnage d'animaux dont nous avons disposé, le poids du gras mésentérique a été exprimé en $\mathrm{g} / 100 \mathrm{~kg}$ de poids vif. L'analyse de variance ne fait apparaître aucune différence significative $(P>0,05)$ entre les lots de mâles et de femelles. La corrélation gras mésentérique-poids corporel permet de montrer que le premier facteur ne rend compte que de $30 \%$ de la variance des données groupées (mâles + femelles), ce qui amène à envisager l'existence de variations saisonnières.

Le poids du gras mésentérique est maximum en automne. Pour les deux années consécutives au cours desquelles des prélèvements ont été effectués, la tendance générale de l'évolution pondérale du gras mésentérique (fig. 1) se caractérise par une décroissance de l'automne (octobre/novembre) à la fin de l'hiver (février/mars). Des valeurs faibles sont encore observées en été.

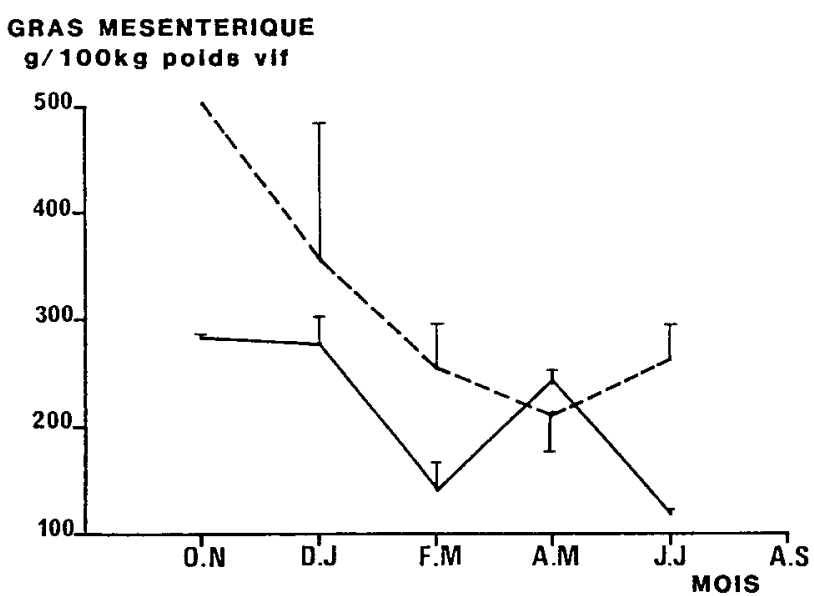

FIG. 1. - Variations, au cours de deux années, du poids frais de tissu gras mésentérique.

La teneur en lipides totaux suit la même évolution (fig. 2). Elle décroît d'environ $40 \%$ à la fin de l'automne-début de l'hiver jusqu'à atteindre moins de $10 \%$ en juin/juillet. La composition en acides gras pour ces deux périodes différentes est présentée dans le tableau 2. La période juin/juillet se caractérise 
par un coefficient d'insaturation significativement $(P<0,05)$ plus élevé qu'au début de I'hiver. La différence tient essentiellement aux proportions d'acide linoléique.

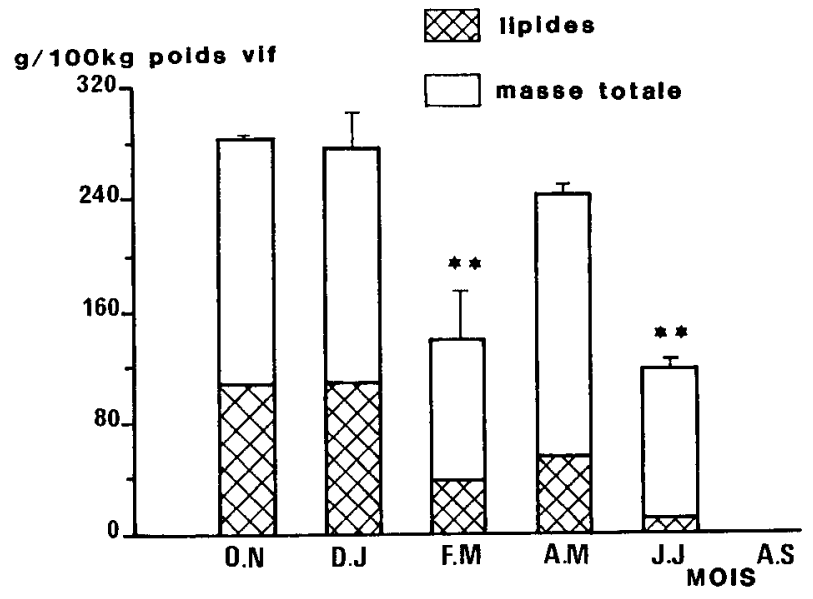

FIG. 2. - Influence de la saison sur la teneur en lipides du gras mésentérique. ** $\mathrm{P}<0,01$.

TABLEAU 2

Composition en acide gras du tissu adipeux mésentérique.

\begin{tabular}{lrr}
\hline \multicolumn{1}{c}{ Paramètre } & Décembre/janvier & \multicolumn{1}{c}{ Juin/juillet } \\
\hline Teneur en lipides (\%) & $39,10 \pm 3,48$ & $8,17 \pm 3,15$ \\
\hline & & \\
Acides gras (\%) & & \\
C14:0 $16: 0$ & $3,88 \pm 0,40$ & $2,01 \pm 0,36$ \\
C16:1 & $3,54 \pm 0,79$ & $31,38 \pm 0,81$ \\
C17:0 & $1,56 \pm 0,54$ & $3,66 \pm 0,04$ \\
C17: $18: 0$ & $0,50 \pm 0,16$ & $1,64 \pm 0,21$ \\
C18:1 & $24,18 \pm 1,64$ & $0,49 \pm 0,13$ \\
C18:2 & $29,00 \pm 5,00$ & $22,24 \pm 2,40$ \\
C18:3 & $4,05 \pm 1,21$ & $7,89 \pm 2,18$ \\
\hline Coefficient & $0,42 \pm 0,17$ & $0,97 \pm 0,45$ \\
d'insaturation & & \\
\hline
\end{tabular}




\section{Paramètres biochimiques plasmatiques.}

Valeurs moyennes. - Les valeurs moyennes des 7 paramètres plasmatiques mesurés sont rassemblées dans le tableau 3. II n'apparaît pas de différence liée au sexe.

TABLEAU 3

Valeurs moyennes $( \pm \mathrm{SEM})$ des paramètres biochimiques plasmatiques.

\begin{tabular}{lcrr}
\hline & $\begin{array}{c}\text { Mâles } \\
(\mathrm{n}=36)\end{array}$ & \multicolumn{1}{c}{$\begin{array}{c}\text { Femelles } \\
(\mathrm{n}=15)\end{array}$} & Moyenne \\
\hline Cholestérol $(\mathrm{mg} / \mathrm{dl})$ & $73 \pm 0,4$ & $80 \pm 3$ & $75 \pm 3$ \\
Glycérol $(\mathrm{mg} / \mathrm{dl})$ & $1,31 \pm 0,15$ & $1,13 \pm 0,22$ & $1,26 \pm 0,14$ \\
Glucose $(\mathrm{mg} / \mathrm{dl})$ & $148 \pm 0$ & $187 \pm 18$ & $158 \pm 8$ \\
Protéines totales $(\mathrm{g} / \mathrm{dl})$ & $9,46 \pm 0,28$ & $9,69 \pm 0,26$ & $9,54 \pm 0,21$ \\
Albumine $(\mathrm{g} / \mathrm{dl})$ & $5,97 \pm 0,21$ & $6,01 \pm 0,30$ & $5,97 \pm 0,17$ \\
Urée $(\mathrm{mg} / \mathrm{dl})$ & $43,6 \pm 2,6$ & $49,0 \pm 4,1$ & $45,3 \pm 2,3$ \\
Créatinine $(\mathrm{mg} / \mathrm{dl})$ & $2,28 \pm 0,10$ & $2,24 \pm 0,11$ & $2,28 \pm 0,08$ \\
\hline
\end{tabular}

Evolution saisonnière. - Elle est présentée à partir des données initiales, non corrigées suivant le poids vif, exprimées par la moyenne et l'erreur standard à chacun des stades (fig. 3 ).

Cholestérol: Les niveaux plasmatiques de cholestérol $(\mathrm{mg} / \mathrm{dl})$ décroissent significativement de l'automne à la fin de l'hiver (octobre/novembre vs février/mars : $P<0,05)$. Le taux le plus bas est observé en mars $(50 \pm 4)$ et la valeur maximum d'automne est atteinte en septembre $(89 \pm 5)$.

Glycérol: II apparaît d'importantes variations individuelles. Néanmoins, l'écart entre les valeurs $(\mathrm{mg} / \mathrm{dl})$ faibles d'octobre/novembre $(0,86 \pm 0,11)$ et le maximum de décembre/janvier $(1,79 \pm 0,24)$ est significatif $(P<0,01)$.

Glucose : La glycémie ( $\mathrm{mg} / \mathrm{dl})$ reste stable tout au long de l'année, les taux mensuels oscillant autour de la moyenne $(158 \pm 8)$.

Protéines totales, albumine: Les protéines plasmatiques sont à leur niveau le plus bas en février; d'une façon générale, leur taux reste faible jusqu'en septembre. L'évolution de l'albuminémie ne montre aucune tendance saisonnière marquée ; les variations du taux de protéines totales semblent donc attribuables essentiellement aux globulines.

FIG. 3. - Evolution des paramètres sanguins (teneurs rapportées au plasma) en fonction de la saison (moyennes significativement différentes des valeurs de la période précédente: ${ }^{*} P<0,05$; $\left.{ }^{*} \mathrm{P}<0,01\right)$.

(n) : valeurs à relativiser du fait d'effectifs $n$ faibles.

$\mathrm{j}$ : moyennes portant sur les jeunes de 8-12 mois. 

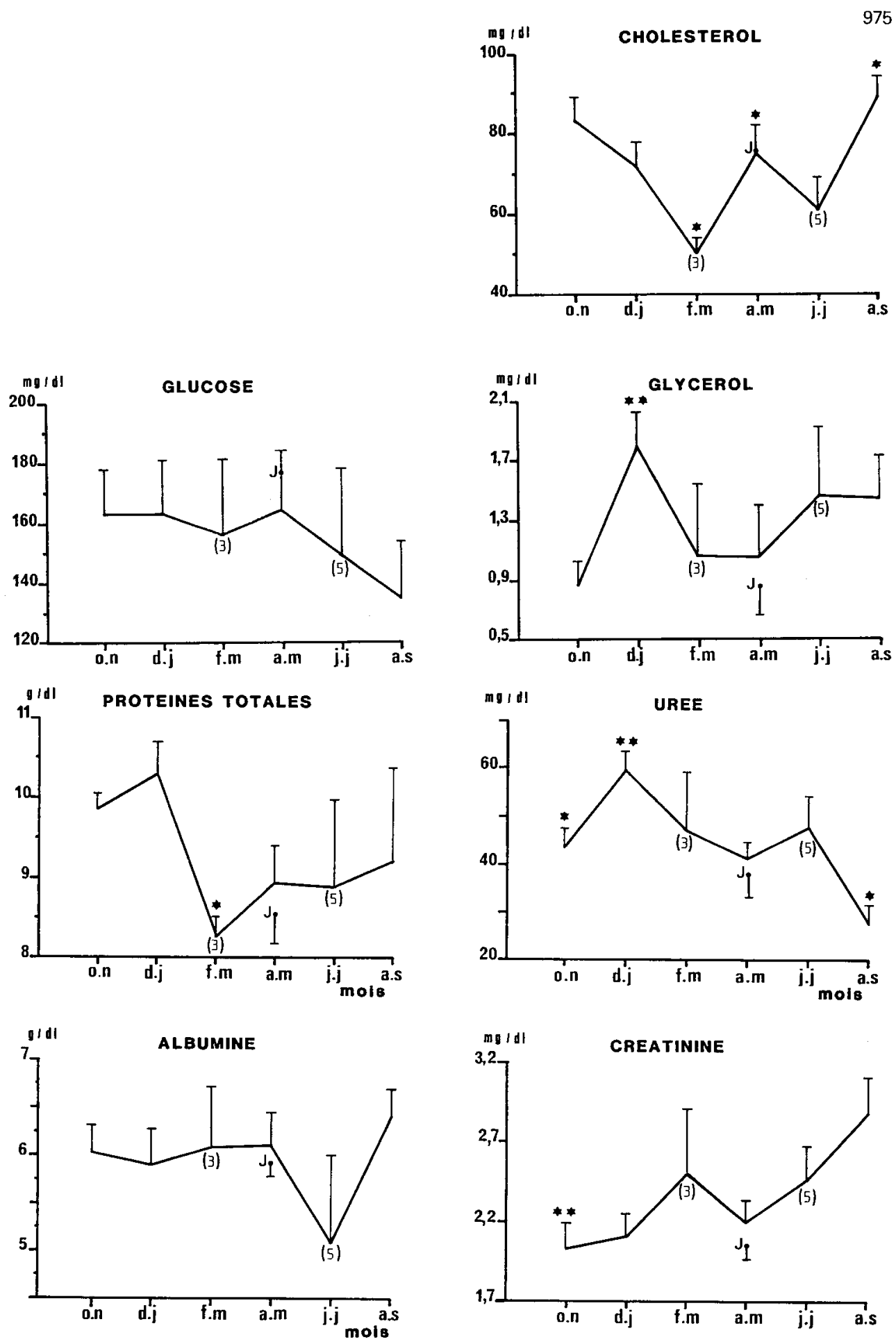

Reproduction, nutrition, développement $n^{\circ} 4 A / 88$. -9 
Urée : L'urémie présente des variations de grande amplitude. Ainsi, les valeurs mesurées $(\mathrm{mg} / \mathrm{dl})$ sont pratiquement deux fois plus élevées en hiver (décembre/janvier : 59,8 \pm 3,8) qu'à la fin de l'été (août/septembre : $27,3 \pm 4,4$ ).

Créatinine: La créatinine $(\mathrm{mg} / \mathrm{dl})$ fluctue significativement au cours de l'année d'un minimum automnal (octobre/novembre : $2,03 \pm 0,16$ ) à un maximum atteint en été (août/septembre : $2,88 \pm 0,23$ ).

\section{Discussion.}

Chez un grand nombre de mammifères sauvages vivant dans les régions tempérées ou nordiques, la croissance pondérale présente un cycle saisonnier nettement marqué (revue in McEwan, 1975), le gain maximum de poids corporel étant le plus souvent observé avant le début de l'hiver. Quelques études font mention de telles variations saisonnières de poids chez des populations naturelles de sangliers (Briedermann, 1970 ; Jezierski et Myrcha, 1975 ; Klein, 1984 ; Pepin et al., 1987). Ces variations sont à mettre en relation avec la production saisonnière de fruits et de graines (glands, faînes) de la forêt. Il a pu être montré récemment, en conditions expérimentales d'élevage, qu'un cycle annuel de la croissance corporelle persistait chez des animaux nourris à volonté (Mauget, 1986). Ces variations saisonnières de poids peuvent être accentuées chez les femelles dont la reproduction et l'allaitement se déroulent vers la fin du printemps, période au cours de laquelle les disponibilités alimentaires décroissent (Aumaitre et al., 1984). Il existerait ainsi un rythme saisonnier interne du métabolisme que traduiraient certains des paramètres que nous avons mesurés dans ce travail.

Ainsi, en l'absence d'autre indice facilement accessible de l'état d'adiposité chez le Sanglier, le gras mésentérique, caractérisé par sa masse ou sa teneur en lipides, peut être considéré comme un bon indice de l'état des réserves adipeuses. Le stockage maximum est réalisé au cours de l'automne et correspond au maximum de poids corporel. Les réserves diminuent ensuite progressivement au cours de l'hiver et du printemps.

Le cholestérol est un des métabolites dont le niveau plasmatique présente les variations saisonnières les plus marquées. Le niveau maximum de cholestérol est concomitant du maximum des réserves adipeuses. Des profils annuels comparables ont été caractérisés chez d'autres ongulés sauvages (Cerf de Virginie : Morris et Bubenik, 1983; Mouflon: Hebert, 1978; Pécari : Lochmiller, 1985). Dans chacun de ces exemples, il a pu être mis en évidence une corrélation positive entre le taux de cholestérol et le niveau alimentaire. Par ailleurs, chez la truie gestante (Walsh Hentges et al., 1987) le taux de cholestérol le plus bas est observé en fin de gestation en situation de restriction alimentaire sévère.

Le glycérol varie en phase inverse du cholestérol. Les valeurs élevées observées en décembre/janvier pourraient être liées à un accroissement de la mobilisation des réserves lipidiques au cours de cette période de faible disponibilité alimentaire et de température externe basse. Les variations saisonnières de la lipolyse décrites chez le Renne (Larsen et al., 1985) peuvent permettre une interprétation dans ce sens. 
Le glucose ne présente aucune variation saisonnière notable. La glycémie moyenne $(158 \mathrm{mg} / \mathrm{dl})$ est comparable aux valeurs déterminées chez d'autres populations sauvages de sangliers (Singer et Ackerman, 1981). Elle paraît supérieure aux références établies chez le porc domestique. II faut souligner que ces valeurs élevées garantissent la qualité et la bonne conservation des échantillons sanguins prélevés.

Des fluctuations cycliques du métabolisme protéique doivent exister, ainsi qu'en témoignent les variations saisonnières des protéines plasmatiques, de l'urée et de la créatinine. Tumbleson (1972) a montré que chez le porc domestique, dans le cas de carence en protéines de la ration, le taux plasmatique des protéines totales était abaissé, l'urémie ne variant pas significativement. Nos données font apparaître qu'au cours de l'hiver, protéines totales et urée présentent la même évolution décroissante alors que le taux de créatinine augmente. Ceci pourrait rendre compte d'une perte musculaire intervenant parallèlement à l'utilisation des réserves lipidiques durant la période hivernale.

En conclusion, cette étude longitudinale a permis de caractériser, sur l'échantillonnage d'animaux dont nous avons pu disposer, l'existence de variations saisonnières du métabolisme chez le Sanglier. Les taux de cholestérol et de créatinine paraissent assez bien refléter les modifications hivernales de l'état nutritionnel. Toutefois, l'interprétation des variations doit rester prudente, compte tenu de la dispersion des valeurs que peuvent entraîner, entre autres, l'échantillonnage (effectifs, âge, sexe), les facteurs individuels, le moment des prélèvements par rapport à la prise de nourriture, le stress lié à la manipulation.

Reçu en décembre 1987. Accepté en mai 1988.

Remerciements. - Nous tenons à remercier les personnels de l'Office National de la Chasse de la Réserve de Chizé qui nous ont facilité la collecte des échantillons. Les remarques et conseils constructifs de $\mathrm{M}$. Etienne nous ont aidés à l'amélioration du manuscrit.

\section{Références}

AUMAITRE A., QUERE J. P., PEINIAU J., 1984. Influence du milieu sur la reproduction hivernale et la prolificité de la laie. Symp. int. Sanglier, Ed. I.N.R.A. Publ., Coll. I.N.R.A. ${ }^{\circ} 22$, pp. 69-78.

BARRAT J., DEVAUD J. P., AUBERT M. F. A., 1984. Paramètres biochimiques et hématologiques, pathologie infectieuse et parasitaire du sanglier sauvage (Sus scrofa). Symp. int. Sang/ier, Ed. I.N.R.A. Publ., Coll. I.N.R.A. n²2, pp. 113-119.

BENJAMINSEN E., DISHINGTON I. W., 1981. Plasma constituents in the sow. Minerals, glucose, urea- $\mathbf{N}$, protein and transaminases in relation to weaning. Acta vet. scand., 22, 1-9.

BRIEDERMANN L., 1970. Zum Körper- und Organwachstum des Wildschweines D.D.R. Arch. Forstwes., 19, 401-420.

DOORNENBALL H., TONG A. K. W., MARTIN A. H., SATHER A. P., 1983 . Studies on the performance, development and carcass composition of the growing pig: effects of sex, feeding regime and age on blood serum parameters. Can. J. anim. Sci., 56, 977-984. 
DOORNENBALL H., TONG A. K. W., SATHER A. P., 1986. Relationships among serum characteristics and performance and carcass traits in growing pigs. J.anim. Sci., 62, 1675-1681.

GIRARD J. P., BOUT J., SALORT D., 1988. Lipides et qualités des tissus adipeux et musculaires de porc ; facteurs de variation. Journées Rech. Porcine en France, 20, 255-278.

HEBERT D., 1978. Blood chemistry as an indicator of nutritional condition in Bighorn sheep. Proc. Northern wild sheep and goat Conf., Pentincton (Canada), pp. 365-387.

JEZIERSKI W., MYRCHA A., 1975. Food requirements of a wild boar population. Pol. ecol. Studies, 1, $61-83$.

KLEIN F., 1984. Contribution à l'étude de la croissance du Sanglier (Sus scrofa) par capture et recapture. Symp. int. Sanglier, Ed. I.N.R.A. Publ., Coll. I.N.R.A. n²2, pp. 55-67.

LARSEN T. S., NILSON N. O., SCHYTTE BLIX A., 1985. Seasonal changes in lipogenesis and lipolysis in isolated adipocytes from Svalbard and Norwegian reindeer. Acta physiol. scand., 123, 97-104.

LOCHMILLER R. L., HELLGREN E. C., VARNER L. W., GREENE L. W., AMOSS M. S., SEAGER S. W. J., GRANT W. E., 1985. Physiological responses of the adult male collared peccary, Tayassu tajacu (Tayassuidae), to severe dietary restriction. Comp. Biochem. Physial., 82A, $59-65$.

LOCHMILLER R. L., HELLGREN E. C., VARNER L. W., GRANT W. E., 1986. Serum and urine biochemical indicators of nutritional status in adult female collared peccaries. Comp. Biochem. Physiol., 83A, 477-488.

MATSCHKE G. H., 1964. The influence of oak mast on European wild hog reproduction. Proc. ann. Conf. South. Game and Fish Com., 18, 35-39.

MATSCHKE G. H., 1967. Aging European wild hogs by dentition. J. Wildl. Manag., 31, 109-113.

MAUGET R., 1986. Seasonal changes in growth and food intake in the wild boar - Endocrine correlates. In I. ASSENMACHER and J.BOISSIN, Endocrine regulations as adaptative mechanisms to the environment. Int. Coll. C.N.R.S., pp. 325-328.

McEWAN E. H., 1975. The adaptative significance of the growth patterns in cervids compared with the other ungulate species. Zool. Zh., 54, 1221-1232.

MORRIS J. M., BUBENIK G. A., 1983. Seasonal levels of minerals, enzymes, nutrients and metabolic products in plasma of intact and castrated adult male white-tailed deer (Odocoileus virginianus). Comp. Biochem. Physiol., 74A, 21-28.

PAYNE J. M., DEW S. M., MANSTON R., FAULKS M., 1970. The use of metabolic profile test in dairy herds. Vet. Rec., 87, 150-158.

PEPIN D., SPITZ F., JANEAU G., VALET G., 1987. Dynamics of reproduction and development of weight in the Wild boar (Sus scrofa) in South-west France. Z. Saügetierk., 52, 21-30.

SEAL U. S., NELSON M. E., MECH L. D., HOSKINSON R. L., 1978. Metabolic indicators of habitat differences in four Minnesota deer populations. J. Wildl. Manag., 42, 746-754.

SINGER F. J., ACKERMAN B. B., 1981. Food availability, reproduction, and condition of European wild boar in Great Smoky National Park. Res. Mgmt Report 43, U.S. Dept int. nat. Park Service.

SMITH M. L., RICK L., SHEPPARD S. T., FARIS B. L., 1978. Reference ovine serum chemistry values. Am. J. vet. Res., 39, 321-323.

TUMBLESON M. E., 1972. Protein-caloric undernutrition in young Sinclair $(\mathrm{S}-1)$ miniature swine : serum biochemic and hematologic values. Technicon int. Congr. New York Technicon ed., $63 \mathrm{pp}$.

URSACHE O., QUINCHON C., TILLON J. P., 1979. Profils biochimiques chez les truies reproductrices. Revue Méd. vét., 130, 1027-1037.

WALSH HENTGES L. S., WILLIAMS A. C., MANGHAM W. A., MARTIN R. J., 1987. Influence of food intake during late gestation on serum lipids of sows and their progeny. Biol. Neonate, 52, 292-300.

WARREN R. J., KIRKPATRICK R. L., OLSCHLAEGER A., GWAZDAUSKAS F. G., 1981. Dietary and seasonal influences on nutritional indices of adult male white-tailed deer. J. Wildl. Manag., 45, 926-936. 